\title{
Clinical findings just after return to play predict hamstring re-injury, but baseline MRI findings do not
}

\author{
Robert-Jan De Vos, ${ }^{1,2,3}$ Gustaaf Reurink, ${ }^{2}$ Gert-Jan Goudswaard, \\ Maarten $\mathrm{H}$ Moen, ${ }^{4,5}$ Adam Weir, ${ }^{1}$ Johannes L Tol ${ }^{1}$
}

Additional material is

published online only. To view please visit the journal online (http://dx.doi.org/10.1136/ bjsports-2014-093737).

${ }^{1}$ Department of Sports Medicine, Aspetar Orthopaedic and Sports Medicine Hospital, Doha, Qatar

${ }^{2}$ Department of Orthopaedics, Erasmus Medical Centre, Rotterdam, The Netherlands ${ }^{3}$ Department of Sports Medicine, The Hague Medical Center, Leidschendam, The Netherlands

${ }^{4}$ Department of Sports Medicine, Bergman Clinics, Naarden, The Netherlands

${ }^{5}$ The Sportsphysician Group, St. Lucas Andreas Hospital, Amsterdam, The Netherlands

\section{Correspondence to} Dr Robert-Jan de Vos, Department of Orthopaedics, Erasmus Medical Centre Room Hs-104, PO Box 2040, Rotterdam 3000 CA, The Netherlands: rj_devos@hotmil.com; r.devos@erasmusmc.nl

R-JDV and $G R$ contributed equally.

Accepted 30 June 2014 Published Online First 18 July 2014

\section{ABSTRACT}

Background Acute hamstring re-injuries are common and hard to predict. The aim of this study was to investigate the association between clinical and imaging findings and the occurrence of hamstring re-injuries.

Methods We obtained baseline data (clinical and MRI findings) of athletes who sustained an acute hamstring injury within 5 days of initial injury. We also collected data of standardised clinical tests within 7 days after return to play (RTP). The number of re-injuries was recorded within 12 months. We analysed the association between the possible predictive variables and re-injuries with a multivariate Cox proportional-hazards regression model.

Results Eighty patients were included at baseline and 64 patients could be included in the final analysis because data after RTP were not available in 16 cases. There were 17 re-injuries (27\%). None of the baseline MRI findings were univariately associated with re-injury. A higher number of previous hamstring injuries (adjusted OR (AOR) 1.33; 95\% Cl 1.11 to 1.61), more degrees of active knee extension deficit after RTP (AOR 1.13; 95\% $\mathrm{Cl} 1.03$ to 1.25), isometric knee flexion force deficit at $15^{\circ}$ after RTP (AOR 1.04; 95\% Cl 1.01 to 1.07) and presence of localised discomfort on hamstring palpation after RTP (AOR 3.95; 95\% Cl 1.38 to 11.37) were significant independent predictors of re-injury. Athletes with localised discomfort on hamstring palpation just after RTP were consequently almost four times more likely to sustain a re-injury.

Conclusions The number of previous hamstring injuries, active knee extension deficit, isometric knee flexion force deficit at $15^{\circ}$ and presence of localised discomfort on palpation just after RTP are associated with a higher hamstring re-injury rate. None of the baseline MRI parameters was a predictor of hamstring re-injury.

Trial registration number ClinicalTrial.gov number NCT01812564.

\section{INTRODUCTION}

High re-injury rates remain a major problem following acute hamstring injuries, despite increasing use of sophisticated imaging techniques, prevention and treatment options. ${ }^{12}$ Hamstring re-injury rate is $14-63 \%$ within 2 years after the initial injury. Re-injuries require longer rehabilitation. ${ }^{1}$

A recent systematic review on risk factors for hamstring re-injury has reported limited evidence for an association with (1) a previous ipsilateral ACL reconstruction, (2) a larger volume measured by MRI and (3) a grade 1 lesion on MRI of the initial injury as predicting recurrence. ${ }^{3}$ Athletes who undertook progressive daily home-based agility and stabilisation exercises were at lower risk for such re-injury. ${ }^{3}$ Although there is no evidence for fibrous tissue formation and reduced hamstring strength as re-injury risk factors, they are frequently mentioned as risk factors. ${ }^{4} 5$ This is thought to result from inadequate rehabilitation, a premature return to play (RTP) or a combination of both. ${ }^{67}$

The timing of RTP is challenging and generally based on expert opinion. Currently there is no consensus on RTP decision-making. ${ }^{8}$ There is one retrospective study comparing different rehabilitation protocols and RTP strategies. ${ }^{9}$ In daily clinical practice an athlete is normally regarded as being fit to RTP if there is a pain-free full range of motion and asymptomatic completion of sports-specific activities. $^{6}{ }^{10}$ Despite this approach, re-injury rates remain high. Ideally, the results of a subjective assessment in combination with radiological and clinical findings would enable the clinician to predict a safe RTP without a high risk of re-injury. However, findings just after RTP have never been described in association with hamstring re-injury.

The aim of this study, a substudy of the Dutch Hamstring Injection Therapy study reported in the New England Journal of Medicine, ${ }^{11}$ is to describe the association between clinical and imaging findings at baseline (including MRI findings of the initial injury) and standardised clinical tests just after RTP with the occurrence of hamstring re-injuries.

\section{METHODS}

\section{Participants}

The patients included in this study were involved in a double-blind randomised controlled trial (RCT) on the effect of platelet-rich plasma in acute hamstring injuries (ClinicalTrial.gov number NCT01812564). ${ }^{11}$ In brief, the study was performed at three sports medicine departments (a large general district hospital, a university hospital and the medical centre of the national football association). Participants received either two injections of $3 \mathrm{~mL}$ of either platelet-rich plasma (Autologous Conditioned Plasma, Biocore, Arthrex Inc, Karlsfeld, Germany) or normal saline at the site of injury. There was no difference in the primary outcome measure (time to RTP) and re-injury rate between these two groups, and therefore it represents a normal cohort. ${ }^{11}$

As outlined in our previous publication, ${ }^{11}$ the eligibility criteria for the present study are age $18-50$ years, a clinical and radiological diagnosis of acute hamstring injury within 5 days from injury, MRI grade 1 or 2 hamstring lesion and the availability of re-assessment within 7 days after RTP. The criteria for a clinical diagnosis of a hamstring muscle 
strain were: acute onset of posterior thigh pain, pain on hamstring stretch and resisted contraction and pain on hamstring muscle palpation. The clinical diagnosis was established by one of the six participating sports medicine physicians. MRI was performed in each participant within 5 days from initial injury, using a $1.5 \mathrm{~T}$ magnet system (Magnetom Essenza, Siemens) and a body matrix coil. MRIs were assessed by a musculoskeletal radiologist to confirm the diagnosis of a hamstring muscle injury. Patients and the supervising physiotherapist were blinded to the severity of the lesion on MRI. Exclusion criteria were a contraindication for MRI, chronic posterior thigh symptoms, persistent chronic low back pain, posterior thigh injury due to extrinsic trauma, inability to perform active rehabilitation, no desire to return to full sports activity, unwillingness to receive intramuscular injections and previous injection therapy for this injury.

At inclusion, informed consent was acquired from all patients. Approval was obtained from the Medical Ethics Committee Zuidwest Holland, Voorburg, The Netherlands.

\section{Procedure}

\section{Baseline patient characteristics at the time of initial injury}

At baseline we recorded age, sex, type of sports, sports activity level (recreational or competitive), type of injury (sprinting type or non-sprinting type) and number of prior ipsilateral and contralateral hamstring injuries. Competitive athletes were individuals who played league matches at the highest levels of their club. A sprinting-type injury was an injury that occurred during a maximum or near maximum sprint. ${ }^{12}$ Injuries that occurred during stretching, deceleration phase of sprinting, high kicking or otherwise not specified were classified as non-sprinting type.

\section{MRI findings at the time of initial injury}

We used standardised scoring forms to assess MRIs at baseline. ${ }^{13}$ We measured the increased T2 signal intensity on the fluid sensitive sequences (short T1 inversion recovery or proton-densityfat-saturated) in craniocaudal, transverse and anteroposterior dimensions. We recorded the longitudinal length (craniocaudal in $\mathrm{cm}$ ) of the lesion and the distance in $\mathrm{cm}$ from the ischial tuberosity. The cross-sectional area was calculated as a percentage of the total muscle cross-sectional area in the transversal plane. The volume of the muscle lesion in $\mathrm{cm}^{3}$ was measured using the formula for a prolate ellipsoid $((\pi / 6) \times$ anteroposterior $\times$ transverse $\times$ craniocaudal extent). ${ }^{14}$ We recorded the involved muscle (s) and performed grading of the injury ${ }^{13}$ : grade 1 increased signal intensity on fluid sensitive sequences without evidence of a macroscopic tear and grade 2 increased signal intensity on fluid sensitive sequences with a partial tear. Good to excellent interobserver and intraobserver reliability was found for these MRI findings used in a previous study. ${ }^{15}$

\section{Rehabilitation programme}

All patients included performed a progressive-phased, criteriabased rehabilitation programme which was based on the best available evidence. ${ }^{6} 16 \quad 17$ The rehabilitation programme consisted of twice-weekly physiotherapist-supervised training sessions combined with daily home-based exercises (see online supplementary table S1). ${ }^{11}$ The home exercise programme consisted of a progressive agility and trunk stabilisation exercise protocol. ${ }^{17}$ The number of supervised physiotherapy sessions and daily home-based exercise sessions were logged. Instructional videos of the exercises were supplied on an openly accessible website. The physiotherapists and patients were instructed to progress through the rehabilitation programme as fast as possible according to the prespecified functional progression criteria. With these instructions, we aimed to stimulate early RTP. However, we also emphasised that criteria were symptom-based, as opposed to time-based progressions.

\section{RTP decision}

Clearance for RTP was given by the supervising physiotherapist once the patient completed the criteria-based rehabilitation programme. According to standardised rehabilitation protocol an athlete was ready to RTP once he or she met the following criteria: symptom-free (eg, pain and stiffness) during: (1) full range of motion; (2) full-speed sprinting and (3) sport-specific movements (such as jumping and cutting). ${ }^{6} 17$ The final phase of the rehabilitation programme consisted of unhindered functional sports-specific testing.

The physiotherapist was blinded to the data of the clinical findings collected by the principal investigator. The physiotherapist and the patient were informed that a lesion was present on the baseline MRI and therefore proved the clinical diagnosis, but they were blinded for the grading and extent of the injury. The principal investigator was not involved in the RTP decision and did not advise patients on RTP decision based on the baseline MRI or clinical findings just after RTP.

\section{Questionnaire and recovery score just after RTP}

All patients included were invited for a re-assessment within 7 days of RTP. The Hamstring Outcome Score (HaOS) was completed, ${ }^{18}{ }^{19}$ consisting of five categories (symptoms, muscle soreness, pain, function in sports and quality of life). The mean score is calculated and displayed between 0\% (lowest score) and $100 \%$ (maximum score). The $\mathrm{HaOS}$ is a screening tool for assessment during normal activities of daily living and sports and used in previous cohorts. ${ }^{18} 19$ For our study, the questionnaire was translated into Dutch by a registrar in Sports Medicine who is a native speaker (GR).

Perceived recovery was measured with a seven-point Likert self-rating scale ranging from 'completely recovered' ( 0 points) to 'worse than ever' (7 points). Complete recovery was considered as a successful outcome. The time to RTP was measured as the number of days from the initial injury until return to full training or match play in the desired sport.

\section{Clinical assessment just after RTP}

The post-RTP clinical evaluation consisted of hamstring flexibility testing, strength testing and muscle palpation. The flexibility of the hamstring muscles was assessed with the active knee extension test ${ }^{20}$ and the passive straight leg raise test. ${ }^{16-18} 20$ Participants were examined in the supine position and an inclinometer was placed on the anterior tibial border. Both the injured and the uninjured legs were tested. For the active knee extension test, participants were positioned with the ipsilateral hip in $90^{\circ}$ flexion. Subsequently, participants were asked to extend the knee until experiencing maximal tolerable stretch, with the contralateral leg fixed flat on the examination table. The maximum absolute knee angle was measured.

For the passive knee extension test participants were instructed to fully relax the leg. Subsequently the leg was lifted by the principal investigator with the hip still in $90^{\circ}$ flexion, and the knee in increasing extension until the maximal tolerable stretch was experienced. The contralateral leg remained flat on the examination table. At the end point of maximal tolerable stretch, the angle between the leg axis and the horizontal examination table was measured. For both tests the absolute flexibility deficit was calculated by subtracting the established angle of the 
injured leg from the uninjured leg. Furthermore, participants were asked whether they experienced normal stretch or localised discomfort in the posterior thigh during the tests.

Isometric knee flexion force was measured using handheld dynamometry. ${ }^{21}$ Participants were tested in prone with a knee flexion angle of $90^{\circ}$ and $15^{\circ}$. Each leg was tested three times in both angles. The principal investigator positioned the dynamometer at the participant's heel and applied force to the heel in upward direction, gradually increasing in 3-5 s. Participants were instructed to resist the force applied by the principal investigator (break test). At the point that the participants were not able to resist the force, the test was terminated and the force level was recorded. For each angle the highest force value was documented. The relative strength deficit was established as a portion of the maximal force value of the injured leg divided by maximal force value of the uninjured leg.

Palpation of the hamstring muscles was performed with the patient in prone with the leg relaxed and neutral hip and knee position. The entire ipsilateral posterior thigh was carefully palpated from the hamstring origin at the ischial tuberosity to the insertions medial at the pes anserinus and lateral at the head of the fibula. The presence of localised discomfort on palpation was recorded as a dichotomous variable (present or absent).

\section{Outcome measures}

The primary outcome measure in this study was the occurrence of a re-injury. Re-injury was defined as acute posterior thigh pain in the index leg within the prospective study follow-up period of 12 months after the initial injury, which caused time loss from training or match play. ${ }^{22}$ All patients were instructed to contact the principal investigator in case of a possible re-injury. We confirmed re-injury based on a telephone interview. The principal investigator took a thorough history and instructed the patient to perform stretching and contraction manoeuvres of the hamstring muscles to identify localised pain on stretch and contraction. All patients in the study were also asked about the occurrence of re-injuries at the standard 6-month and 12-month follow-ups.

\section{Statistical analysis}

We performed statistical analyses with SPSS software (V.20.0; SPSS, Chicago, Illinois, USA). Descriptive statistics were used to analyse baseline patient characteristics. If the data were normally distributed they were presented as means with a SDs, otherwise median and IQRs are used. To aid in data interpretation, a number of variables were categorised.

We analysed the association between the possible predictor variables and re-injuries with a Cox proportional-hazards regression model. In this model the time (days) from RTP to the event (re-injury) or the end of the follow-up is the main variable. All patients who were available for examination shortly after RTP were included in the final analysis. We excluded patients from the analysis if we could not obtain RTP measurements, because cases with missing values are routinely excluded from the multivariate analysis. Participants that sustained another severe injury (defined as absence from training and matches $>28$ days $^{1}{ }^{23}$ ) during follow-up that was not considered a hamstring re-injury were censored at the time of this injury. Participants lost to follow-up were censored at the time of their last available follow-up.

We first analysed the association between predictor variables and re-injuries in a univariate model. Variables with a $p$ value of $<0.1$ were analysed in a multivariate stepwise regression. We considered a $\mathrm{p}$ value $<0.05$ statistically significant.

\section{RESULTS}

\section{Participants}

One-hundred sixty-one patients were assessed for eligibility and 80 patients were included in the RCT. Finally, 64 patients were available for inclusion in the analysis. In 16 cases (20\%) data just after RTP were not available. The number of re-injuries did not differ significantly between the patients included and excluded from the analysis $(p=0.49$, see table 1$)$. The progress of patients in the study is displayed in a flow chart (figure 1). We performed measurements at a median of 3 days (IQR 2-5) after the RTP date. The characteristics of the patients included and excluded are presented in table 1.

\section{Descriptive measurements at baseline and just after RTP}

The results of the clinical and imaging findings at baseline and just after RTP are displayed in table 2. Twenty-eight per cent of the patients sustained a grade 1 injury and $72 \%$ a grade 2 injury as graded on MRI. In $88 \%$ of the patients a biceps femoris long head injury was present and in $12 \%$ a semitendinosus/semimembranosus injury.

\section{Table 1 Patient characteristics $(n=80)$}

\begin{tabular}{|c|c|c|}
\hline & $\begin{array}{l}\text { Included } \\
(n=64)\end{array}$ & $\begin{array}{l}\text { Excluded } \\
(n=16)\end{array}$ \\
\hline Median age (IQR) & $28(23-33)$ & $28(22-32)$ \\
\hline Gender male/female & $61 / 3$ & $15 / 1$ \\
\hline \multicolumn{3}{|l|}{ Sports } \\
\hline Football & 45 & 11 \\
\hline Futsal (indoor football) & 1 & 0 \\
\hline Field hockey & 11 & 1 \\
\hline Athletics & 4 & 0 \\
\hline Tennis & 1 & 0 \\
\hline American football & 1 & 2 \\
\hline Fitness & 1 & 1 \\
\hline Cricket & 0 & 1 \\
\hline \multicolumn{3}{|l|}{ Level of sports } \\
\hline Competitive & 49 & 10 \\
\hline Recreational & 15 & 6 \\
\hline Median days (IQR) to initial presentation & $3(2-4)$ & $3(2-4)$ \\
\hline $\begin{array}{l}\text { Median number(IQR) of previous hamstring } \\
\text { injuries }\end{array}$ & $1(0-3)$ & $1(0-3)$ \\
\hline \multicolumn{3}{|l|}{ Severity of injury on MRI } \\
\hline Grade 1 & 18 & 5 \\
\hline Grade 2 & 46 & 11 \\
\hline \multicolumn{3}{|l|}{ Involved muscles } \\
\hline Biceps femoris & 56 & 13 \\
\hline Semitendinosus/semimembranosus & 8 & 3 \\
\hline $\begin{array}{l}\text { Mean (SD) longitudinal length (craniocaudal) on } \\
\text { MRI }\end{array}$ & $11.4(5.7)$ & $14.6(6.9)$ \\
\hline $\begin{array}{l}\text { Mean (SD) distance from the ischial tuberosity } \\
\text { on MRI }\end{array}$ & $15(7.8)$ & $14.8(7.7)$ \\
\hline Mean (SD) cross-sectional area on MRI & $37 \%(28)$ & $33 \%(21)$ \\
\hline $\begin{array}{l}\text { Mean (SD) volume of the muscle lesion } \\
\text { on MRI }\left(\mathrm{cm}^{3}\right)\end{array}$ & $285(302)$ & $486(677)$ \\
\hline Median (IQR) time to RTP (days) & $40(31-55)$ & $\begin{array}{l}46(33-67) \\
(n=11)\end{array}$ \\
\hline $\begin{array}{l}\text { Median days (IQR) between RTP and clinical } \\
\text { findings }\end{array}$ & $3(2-5)$ & NA \\
\hline Number of re-injuries & $17(27 \%)$ & $4(36 \%)(n=11)$ \\
\hline
\end{tabular}


Figure 1 Progress of patients through the study (RTP, return to play).
161 patients assessed for eligibility by principal investigator

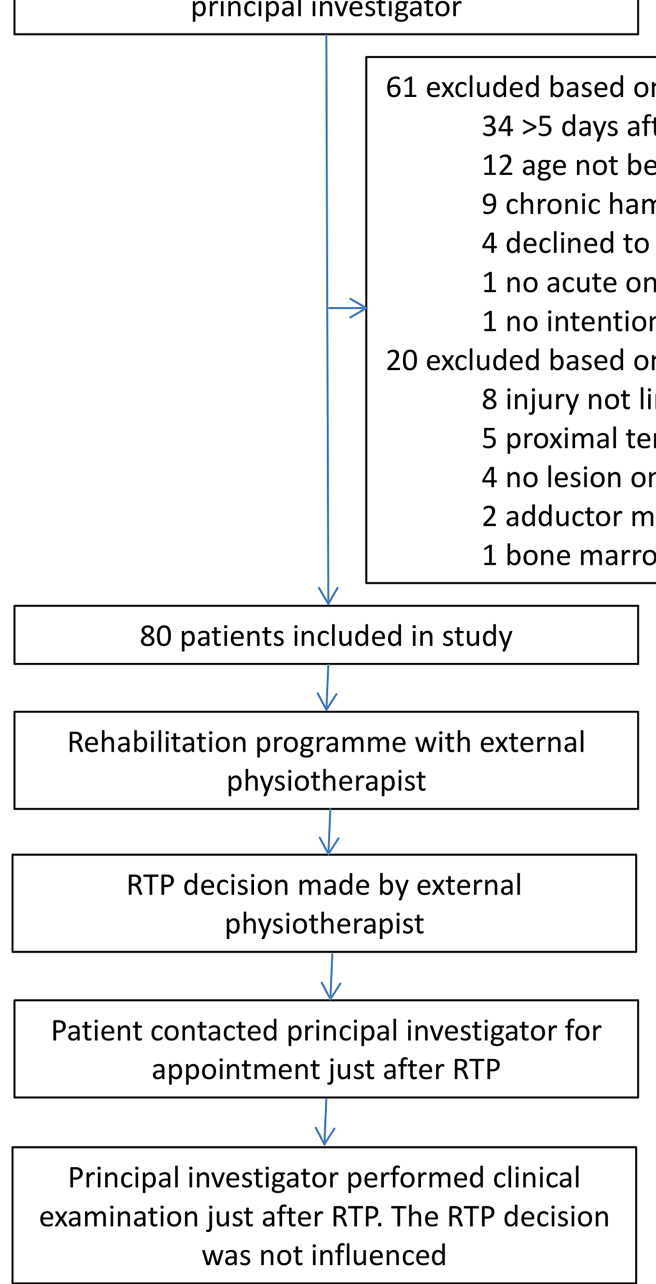

Just after RTP, $66 \%$ of the patients reported a 'complete recovery' on the Likert scale. Discomfort during hamstring flexibility, hamstring resistance testing or on localised palpation was present in $25 \%$ of cases when the independent principal investigator tested those athletes who had been cleared to RTP.

In total, $50 \%$ of the patients kept their daily logs of the rehabilitation programme and returned their logbook. The mean percentage adherence for the supervised physiotherapy programme was $80 \%$. The mean percentage adherence for the home exercise programme was $64 \%$. Owing to these missing data, we refrained from including adherence in the Cox proportional hazards regression model.

\section{Prognostic factors for re-injury}

None of the 64 patients were lost to follow-up during the 12-month follow-up period. There were 17 (27\%) re-injuries. The re-injuries occurred at a median (IQR) of 100 (6-138) days 
Table 2 Descriptive statistics of clinical findings just after RTP

\begin{tabular}{ll}
\hline Clinical test & $\begin{array}{l}\text { Measurement } \\
\text { just after RTP }\end{array}$ \\
\hline Mean (SD) HaOS (0-100) & $88(12)$ \\
Median (IQR) Likert self-rating scale (0-7) & $0(0-1)$ \\
Active knee extension test & \\
$\quad$ Mean (SD) deficit in angle (degrees) & $-2(5)$ \\
Discomfort (present/absent) & $1 / 63$ \\
Passive straight leg raise test & \\
Mean (SD) deficit in angle (degrees) & $-1(4)$ \\
Discomfort (present/absent) & $1 / 63$ \\
Isometric knee flexion resistance in 90 & \\
Mean (SD) force deficit (Newton) & $2(15)$ \\
Discomfort (present/absent) & $8 / 56$ \\
Isometric knee flexion resistance in 15 & \\
Mean (SD) force deficit (Newton) & $1(16)$ \\
Discomfort (present/absent) & $8 / 56$ \\
Localised discomfort on palpation hamstring & $16 / 48$ \\
muscles & \\
(present/absent) & \\
\hline HaOS, Hamstring Outcome Score; RTP, return to play.
\end{tabular}

after RTP. There were 7 (11\%) re-injuries within the first 2 months.

The number of previous hamstring injuries $(\mathrm{p}=0.006)$, presence of discomfort during isometric knee flexion resistance test at $15^{\circ}(\mathrm{p}=0.008)$ and localised discomfort on hamstring palpation $(\mathrm{p}=0.008)$ were univariately associated with hamstring injury recurrence. For the baseline MRI findings examined we did not find significant univariate associations, as well as for all other measured variables. The results of the univariate analysis are shown in table 3 .

Five variables (number of previous hamstring injuries, active knee extension deficit, isometric knee flexion force deficit at $15^{\circ}$, presence of discomfort during isometric knee flexion resistance test at $15^{\circ}$ and localised discomfort on posterior thigh palpation) were included in the multivariate model based on a $\mathrm{p}$ value $<0.1$ in the univariate analysis (table 4 ).

The multivariate model showed that athletes with a higher number of previous ipsilateral and/or contralateral hamstring injuries (adjusted OR (AOR) 1.33; 95\% CI 1.11 to 1.61), more degrees of active knee extension deficit (AOR 1.13; 95\% CI 1.03 to 1.25 ), isometric knee flexion force deficit at $15^{\circ}$ (AOR 1.04; $95 \%$ CI 1.01 to 1.07) and presence of localised discomfort on hamstring palpation (AOR 3.95; 95\% CI 1.38 to 11.37) were more likely to have a re-injury. The presence of discomfort during isometric knee flexion resistance test at $15^{\circ}$ was not a significant independent predictor of re-injury (AOR 2.66; 95\% CI 0.83 to 8.55$)$.

\section{DISCUSSION}

Hamstring re-injury ${ }^{3}$ may have a different set of risk factors to primary hamstring injury. ${ }^{24}$ We evaluated clinical and MRI findings to identify factors associated with re-injury following hamstring injury in competitive and recreational athletes. We evaluated the athletes just after the initial injury and just after RTP, when they successfully completed a criteria-based rehabilitation programme. Some players exhibited a deficit in several functional tests after RTP. Four specific clinical findings that had not fully returned to normal-as compared to the unaffected side-just after RTP appear to be associated with an increased re-injury risk (a higher number of previous hamstring injuries, more degrees of active knee extension deficit, isometric knee flexion force deficit at $15^{\circ}$ and the presence of localised discomfort on posterior thigh palpation just after RTP).

\section{Independent predictors of re-injury which can be used in daily clinical practice}

Significant independent predictors of hamstring re-injury were a higher number of previous hamstring injuries, more degrees of active knee extension deficit, isometric knee flexion force deficit at $15^{\circ}$ and the presence of localised discomfort on posterior thigh palpation just after RTP. These various independent predictors were analysed using a multivariate model. These results provide useful prognostic information for clinicians involved in the treatment of hamstring injuries and who are responsible for the RTP decision.

These findings are important as acute hamstring injuries frequently result in a recurrence with a prolonged rehabilitation time. With easy-to-assess clinical evaluation-performed by clinicians or physiotherapists-those participants with an increased re-injury risk can be identified. These findings emphasise that it is of major importance to monitor the athlete in the first week after RTP and not only at RTP.

We found that athletes with localised discomfort on hamstring palpation just after the RTP date were almost four times more likely to sustain a re-injury compared with athletes with absence of discomfort on palpation. Also, the continuous variables number of previous hamstring injuries (AOR 1.33, 33\% increased risk per number of previous hamstring injury), degrees of active knee extension deficit (AOR 1.13, 13\% increased risk per deficit in degree) and isometric knee flexion force deficit at $15^{\circ}$ (AOR 1.04, 4\% increased risk per deficit in Newton) showed to be of prognostic value. For example, an athlete is at $33 \%$ more risk for re-injury if there is one previous hamstring injury and at $77 \%$ more risk (HR increases with $1.33 \times 1.33=1.77)$ if there are two previous hamstring injuries, compared with no previous hamstring injury.

\section{MRI findings at baseline is not a predictor of re-injury- comparison with previous studies}

Clinical findings just after RTP have not been related to hamstring re-injury rate in previous studies. A recent study from our group described the MRI findings just after RTP. ${ }^{13}$ We reported that in $89 \%$ of clinically recovered hamstring injuries, increased intramuscular signal intensity on fluid sensitive sequences was observed just after RTP. ${ }^{13}$ The presence of increased signal intensity was not discriminative as a predictor for re-injury. The number of re-injuries was too small to draw conclusions on the effect of the presence of fibrous tissue on MRI. These results emphasise that clinical and functional tests seem to be better associated with re-injury rates than findings on MRI just after RTP. $^{10} 25$

In a previous study, Warren $e t a l^{26}$ reported on the value of baseline history and clinical signs measured within 0-3 days in 59 athletes who sustained an acute hamstring injury. They concluded that hamstring injury in the previous medical history predicted re-injury (relative risk of 19.5 and $95 \%$ CI 1.5 to 261.1). Baseline clinical findings for hamstring flexibility, pain on resistance and palpation at the site of the injury were also included, but were found not to be significant early predictors. A comparable study in 30 athletes with acute hamstring injury did not show an association with re-injury and the baseline clinical findings assessed at $12-18 \mathrm{~h}$ postinjury. ${ }^{27}$ The lack of association between clinical test in the acute phase and re-injury risk might 
Table 3 Univariate results of the association between the clinical findings just after RTP and event of re-injuries

\begin{tabular}{|c|c|c|c|}
\hline Variable & $\mathbf{n}$ & HR $(95 \% \mathrm{Cl})$ & $\mathrm{p}$ Value \\
\hline \multicolumn{4}{|l|}{ Patient characteristics } \\
\hline \multicolumn{4}{|l|}{ Categorical variables } \\
\hline Previous hamstring injury & & $2.9(0.8$ to 10.1$)$ & 0.094 \\
\hline No (reference) & 23 & & \\
\hline Yes & 41 & & \\
\hline Previous ipsilateral hamstring injury & & $1.8(0.7$ to 5.0$)$ & 0.233 \\
\hline No (reference) & 30 & & \\
\hline Yes & 34 & & \\
\hline Previous hamstring injury within 12 months & & $1.6(0.6$ to 4.3$)$ & 0.309 \\
\hline No (reference) & 45 & & \\
\hline Yes & 19 & & \\
\hline Previous ipsilateral hamstring injury within 12 months & & 1.6 (0.6 to 4.2$)$ & 0.388 \\
\hline No (reference) & 47 & & \\
\hline Yes & 17 & & \\
\hline Level of sport & & $2.5(0.6$ to 11$)$ & 0.218 \\
\hline Recreational (reference) & 15 & & \\
\hline Competitive & 49 & & \\
\hline \multicolumn{4}{|l|}{ Continuous variables } \\
\hline Age & & $1.00(0.94$ to 1.07$)$ & 0.952 \\
\hline Number of previous hamstring injuries & & 1.26 (1.07 to 1.48$)$ & 0.006 \\
\hline \multicolumn{4}{|l|}{ Injury characteristics } \\
\hline \multicolumn{4}{|l|}{ Categorical variables } \\
\hline Sprinting injury type & & $1.9(0.5$ to 6.5$)$ & 0.321 \\
\hline No (reference) & 17 & & \\
\hline Yes & 47 & & \\
\hline Injured muscle & & $0.5(0.1$ to 3.4$)$ & 0.440 \\
\hline Biceps femoris (reference) & 56 & & \\
\hline Semitendinosus/semimembranosus & 8 & & \\
\hline MRI grade & & $1.3(0.4$ to 4.1$)$ & 0.624 \\
\hline Grade I (reference) & 18 & & \\
\hline Grade II & 46 & & \\
\hline \multicolumn{4}{|l|}{ Continuous variables } \\
\hline Time to RTP & & $0.98(0.94$ to 1.01$)$ & 0.126 \\
\hline MRI hyperintensity volume & & $1.00(1.00$ to 1.00$)$ & 0.112 \\
\hline MRI cross-sectional area (\% of total muscle) & & $0.95(0.18$ to 5.11$)$ & 0.947 \\
\hline MRI craniocaudal length & & $1.00(0.99$ to 1.01$)$ & 0.525 \\
\hline MRI distance to tuber & & $1.00(0.98$ to 1.00$)$ & 0.978 \\
\hline \multicolumn{4}{|l|}{ Characteristics just after RTP } \\
\hline \multicolumn{4}{|l|}{ Categorical variables } \\
\hline Subjective complete recovery & & $0.5(0.2$ to 1.3$)$ & 0.136 \\
\hline Yes (reference) & 22 & & \\
\hline No & 42 & & \\
\hline Localised discomfort on palpation & & 3.7 (1.4 to 9.6$)$ & 0.008 \\
\hline No (reference) & 48 & & \\
\hline Yes & 16 & & \\
\hline Active knee extension discomfort & & 0.1 (0 to 303265.6$)$ & 0.704 \\
\hline No (reference) & 63 & & \\
\hline Yes & 1 & & \\
\hline Passive straight leg raise discomfort & & $2.2(0.3$ to 16.7$)$ & 0.445 \\
\hline No (reference) & 62 & & \\
\hline Yes & 2 & & \\
\hline Discomfort on isometric knee flexion resistance in $15^{\circ}$ & & $3.7(1.4$ to 9.6$)$ & 0.008 \\
\hline No (reference) & 56 & & \\
\hline Yes & 8 & & \\
\hline \multicolumn{4}{|l|}{ Continuous variables } \\
\hline $\mathrm{HaOS}$ & & $0.98(0.95$ to 1.01$)$ & 0.198 \\
\hline Active knee extension deficit & & $1.10(1.00$ to 1.24$)$ & 0.059 \\
\hline Passive straight leg raise deficit & & $0.95(0.85$ to 1.07$)$ & 0.376 \\
\hline Isometric knee flexion force deficit in $15^{\circ}$ & & $1.03(1.00$ to 1.06$)$ & 0.074 \\
\hline
\end{tabular}

HaOS, Hamstring Outcome Score; RTP, return to play. 
Table 4 Independent predictors of re-injuries

\begin{tabular}{lll}
\hline Clinical test & $\begin{array}{l}\text { Adjusted HR } \\
\text { (95\% CI) }\end{array}$ & $\mathbf{p}$ Value \\
\hline $\begin{array}{l}\text { Number of previous hamstring muscle injuries } \\
\text { Active knee extension deficit }\end{array}$ & $1.33(1.11$ to 1.61) & 0.002 \\
$\begin{array}{l}\text { Isometric knee flexion resistance discomfort in } 15^{\circ} \\
\text { Isometric knee flexion force deficit in } 15^{\circ}\end{array}$ & $2.66(0.83$ to 8.55$)$ & 0.100 \\
$\begin{array}{l}\text { Localised discomfort on palpation hamstring } \\
\text { muscles }\end{array}$ & $3.95(1.01$ to 1.07) & 0.020 \\
\hline
\end{tabular}

be explained by the fact that almost all these patients experience pain at the early stages of the injury and therefore the discriminative power of these tests are not yet present in the acute stage. For this reason-and also because it is common in clinical practice-we repeated the assessment of clinical findings just after the RTP moment.

The predictive value of baseline MRI findings of the initial injury for re-injury has been reported in previous studies. One study $^{28}$ did not show an association between the size of the initial injury and re-injury rate. They found, however, that a higher frequency of re-injury was reported in grade 1 hamstring injury at the initial trauma, compared with MRI negative injuries. In a study of 30 Australian Rules Footballers, Verrall and colleagues $^{27}$ found that baseline MRI features were not associated with increased recurrent injury risk within the same playing season. Larger transverse size and volume of injury on MRI was reflected in re-injury risk if the subsequent playing season was also included in the analysis. Koulouris et al ${ }^{29}$ performed a cohort study in 41 Australian Football players and showed a correlation between length of the lesion on MRI and re-injury risk.

Owing to the above mentioned study results, it is suggested that a more severe injury on MRI might result in a longer expected recovery time and postponed RTP decision. In our study, patients were blinded for the severity of the injury on baseline MRI; only the presence of a lesion was confirmed. The RTP decision was therefore not influenced by the grading of the lesion on MRI. We did not find any of the baseline MRI findings to be associated with re-injury. As $89 \%$ of the MRIs still showed abnormalities at RTP, ${ }^{13}$ MRI at RTP did not provide stronger associations (data not shown). Based on our study-in which blinding of the patients and care-providers was performed-baseline MRI is not a predictor of hamstring re-injury.

\section{Study strengths and limitations}

The strength of this study is that one single independent principal investigator performed all clinical tests. He was not involved in the RTP decision and only assessed the clinical findings once the player returned to play. The independent physiotherapistwho made the RTP decision-was blinded to the outcomes of the clinical tests performed by the principal investigator. This allows for the opportunity to study the effects of some of these variables that were tested but not included in the RTP decisionmaking algorithm.

There are also some study limitations. First, the number of patients was relatively low with a subsequent low number of re-injuries. Therefore, the study might be underpowered to provide a definite answer on the effect size of the clinical predictors for re-injury at RTP. A previous article reports that 20-50 cases are needed to detect moderate to strong associations. ${ }^{30}$ In our study, 17 re-injuries were found, meaning that only strong associations could probably be detected. The sample size of the original study was based on the primary outcome (days between injury and RTP) of the RCT. ${ }^{11}$ Therefore, small associations are less likely to be detected with our study. However, the clinical relevance of finding small associations is also questionable.

Furthermore, not all athletes $(n=16,20 \%)$ had clinical examination performed after RTP, which may have resulted in risk of bias. However, the percentage of re-injuries did not differ between the patients included and excluded from the analysis. It was challenging to evaluate all patients shortly after the RTP date and there were athletes who did not achieve RTP due to other injuries. To minimise the risk of bias, these athletes were excluded from the analysis. Two athletes sustained a re-injury after RTP before we could do a clinical assessment and therefore, there may be bias because the outcome (re-injury) resulted in missing data. Another potential source of bias is the fact that re-injuries were diagnosed with a telephone interview. This might have resulted in an overestimation of the re-injuries since posterior thigh pain after an initial hamstring injury is not always due to a hamstring re-injury. However, the principal investigator aimed to collect as much data as possible to establish the diagnosis of a re-injury.

Not all clinical tests we performed have well-examined reliability or validity. The active and passive knee extension tests have good intertester reliability. ${ }^{20}$ For the other clinical tests these characteristics are unknown. Especially discomfort on palpation is difficult to standardise, as pressure of the palpating fingers might influence the results. To prevent large variability in test results, all clinical examinations were performed by one single trained principal investigator. He was not blinded to the side of the injury during the clinical assessment after RTP, which potentially might have led to bias. As this clinical examination was performed within 5 days after RTP (range 2-5), it remains unknown if recorded abnormalities were present at the moment of RTP decision-making or developed within the 2-5 days after RTP.

Last the independent treating physiotherapists were advised to use the standardised rehabilitation protocol; however, they did not $\log$ the actual rehabilitation performed. Variations on the protocol might have been used; although the physiotherapists were instructed to follow the protocol. The amount of bias for daily clinical practice is questionable, as many different protocols and RTP decision criteria are described in literature. ${ }^{8} 31$ The observation that the re-injuries occurred at a median (IQR) of $100(6-138)$ days after RTP suggest that the re-injuries were not a consequence of poor RTP decision-making by the supervising physiotherapists. Furthermore, in our study $27 \%$ of the athletes had a re-injury, which is within the normal range of re-injury rate in the scientific literature (14-63\%). ${ }^{3}$

\section{Recommendations for future studies}

Re-injury prevention could be a focus in future studies. We found that a higher number of previous hamstring injuries, more degrees of active knee extension deficit, isometric knee flexion force deficit at $15^{\circ}$ and the presence of localised discomfort on posterior thigh palpation were associated with higher re-injury risk after patients completed a criteria-based rehabilitation programme. These factors might be implemented in criteria-based rehabilitation programmes. The associated factors can also be used to test this clinical prediction rule in a prospective study. If they are found to hold up to this scrutiny then an intervention study could be performed to see if more stringent RTP criteria results in lower recurrence rates. 
Re-injury prevention might be another interesting focus in future studies. In future research, rehabilitation programmes could be more focused on recovery of the clinical findings that are associated with re-injury. If the re-injury rate in these identified high-risk athletes can be reduced with prevention programmes, it will potentially have a major influence on the number needed to treat.

\section{CONCLUSION}

A higher number of previous hamstring injuries, more degrees of active knee extension deficit, isometric knee flexion force deficit at $15^{\circ}$ and the presence of localised discomfort on posterior thigh palpation after RTP were associated with hamstring re-injury in athletes who completed a criteria-based rehabilitation programme. These factors could be taken into account by clinicians when making an RTP decision and monitoring athletes after RTP. Based on this study, none of the baseline MRI parameters was a predictor of hamstring re-injury.

\section{What are new findings?}

- Clinical findings within 5 days after return to play (RTP) are most valuable for estimating the re-injury risk.

- The number of previous hamstring injuries, active knee extension deficit, isometric knee flexion force deficit at $15^{\circ}$ and presence of localised palpation discomfort after RTP are significantly associated with hamstring re-injury.

- Baseline MRI findings of the initial injury (injured muscle, grade I or II, hyperintensity volume, cross-sectional area, craniocaudal length and distance to tuber) are not associated with the occurrence of hamstring re-injury.

\section{How might it impact on clinical practice in the near future?}

- These study results provide useful prognostic information for clinicians involved in the treatment of hamstring injuries and who are responsible for the return to play (RTP) decision. With positive clinical findings in patients who are subjectively recovered after a standardised rehabilitation and RTP, athletes at risk for hamstring re-injuries can be identified.

- Future studies can focus on the impact of postponed RTP decision and prevention programmes in high-risk athletes. Rehabilitation programmes could focus more on recovery of the clinical findings that are associated with re-injury. If the re-injury rate in these identified high-risk athletes can be reduced with re-injury prevention programmes, it will potentially have a major influence.

Acknowledgements The authors thank the medical staff of The Hague Medical Centre, Medical Centre of the Royal Dutch Football Association and University Medical Centre Utrecht for their contribution to the study. The authors thank Mario Maas for his efforts in the MRI data collection.

Contributors GR designed the study, monitored data collection, analysed and interpreted the data and revised the paper. RV analysed and interpreted the data and drafted the paper, GG interpreted the data and revised the paper. JT designed the study, interpreted the data and revised the paper. MM, AW and JV interpreted the data and revised the paper. All authors gave final approval for the version to be published.

Funding The randomised controlled trial was supported by the Royal Dutch Football Association and Arthrex Medizinische Instrumente $\mathrm{GmbH}$.

Competing interests None.

Ethics approval Medical Ethics Committee Zuidwest Holland, Voorburg, the Netherlands.

Provenance and peer review Not commissioned; externally peer reviewed.

\section{REFERENCES}

1 Ekstrand J, Hägglund $M$, Waldén $M$. Epidemiology of muscle injuries in professional football (soccer). Am J Sports Med 2011;39:1226-32.

2 Ekstrand J, Hägglund $M$, Kristenson $\mathrm{K}$, et al. Fewer ligament injuries but no preventive effect on muscle injuries and severe injuries: an 11-year follow-up of the UEFA Champions League injury study. Br J Sports Med 2013;47:732-7.

3 De Visser HM, Reijman M, Heijboer MP, et al. Risk factors of recurrent hamstring injuries: a systematic review. Br J Sports Med 2012;46:124-30.

4 Silder A, Heiderscheit BC, Thelen DG, et al. MR observations of long-term musculotendon remodeling following a hamstring strain injury. Skeletal Radiol 2008;37:1101-9.

5 Silder A, Reeder SB, Thelen DG. The influence of prior hamstring injury on lengthening muscle tissue mechanics. J Biomech 2010;43:2254-60.

6 Heiderscheit BC, Sherry MA, Silder A, et al. Hamstring strain injuries: recommendations for diagnosis, rehabilitation, and injury prevention. J Orthop Sports Phys Ther 2010;40:67-81.

7 Orchard J, Best TM. The management of muscle strain injuries: an early return versus the risk of recurrence. Clin J Sport Med 2002;12:3-5.

8 Orchard J, Best TM, Verrall GM. Return to play following muscle strains. Clin J Sport Med 2005;15:436-41.

9 Heiser TM, Weber J, Sullivan G, et al. Prophylaxis and management of hamstring muscle injuries in intercollegiate football players. Am J Sports Med 1984;12:368-70.

10 Mendiguchia J, Brughelli M. A return-to-sport algorithm for acute hamstring injuries. Phys Ther Sport 2011;12:2-14.

11 Reurink G, Goudswaard G, Moen M, et al. Platelet-rich plasma injections in acute muscle injury. N Engl J Med 2014;370:2546-7.

12 Askling CM, Malliaropoulos N, Karlsson J. High-speed running type or stretching-type of hamstring injuries makes a difference to treatment and prognosis. Br J Sports Med 2012;46:86-7.

13 Reurink G, Goudswaard G, Tol J, et al. MRI observations at return to play of clinically recovered hamstring injuries. Br J Sports Med 2014;48:1370-6.

14 Slavotinek JP, Verrall GM, Fon GT. Hamstring injury in athletes: using MR imaging measurements to compare extent of muscle injury with amount of time lost from competition. AJR Am J Roentgenol 2002;179:1621-8.

15 Hamilton B, Whiteley R, Almusa E, et al. Excellent reliability for MRI grading and prognostic parameters in acute hamstring injuries. Br J Sports Med 2014;48:1385-7

16 Mason DL, Dickens V, Vail A. Rehabilitation for hamstring injuries. Cochrane Database Syst Rev 2007;(1):CD004575.

17 Sherry MA, Best TM. A comparison of 2 rehabilitation programs in the treatment of acute hamstring strains. J Orthop Sports Phys Ther 2004;34:116-25.

18 Engebretsen $\mathrm{AH}$, Myklebust $\mathrm{G}$, Holme I, et al. Intrinsic risk factors for hamstring injuries among male soccer players: a prospective cohort study. Am J Sports Med 2010;38:1147-53.

19 Engebretsen AH, Myklebust G, Holme I, et al. Prevention of injuries among male soccer players: a prospective, randomized intervention study targeting players with previous injuries or reduced function. Am J Sports Med 2008;36:1052-60.

20 Reurink G, Goudswaard GJ, Oomen HG, et al. Reliability of the active and passive knee extension test in acute hamstring injuries. Am J Sports Med 2013;41:1757-61.

21 Landes S, Nyland J, Elmlinger B, et al. Knee flexor strength after ACL reconstruction: comparison between hamstring autograft, tibialis anterior allograft, and non-injured controls. Knee Surg Sports Traumatol Arthrosc Off J ESSKA 2010;18:317-24.

22 Fuller CW, Ekstrand J, Junge $A$, et al. Consensus statement on injury definitions and data collection procedures in studies of football (soccer) injuries. $\mathrm{Br}$ J Sports Med 2006;40:193-201.

23 Hägglund $M$, Waldén $M$, Ekstrand J. Previous injury as a risk factor for injury in elite football: a prospective study over two consecutive seasons. Br J Sports Med 2006:40:767-72

24 Freckleton G, Pizzari T. Risk factors for hamstring muscle strain injury in sport: a systematic review and meta-analysis. Br J Sports Med 2013;47:351-8.

25 Thorborg K. Why hamstring eccentrics are hamstring essentials. Br J Sports Med 2012;46:463-5.

26 Warren P, Gabbe BJ, Schneider-Kolsky M, et al. Clinical predictors of time to return to competition and of recurrence following hamstring strain in elite Australian footballers. Br J Sports Med 2010;44:415-19. 
27 Verrall GM, Slavotinek JP, Barnes PG, et al. Assessment of physical examination and magnetic resonance imaging findings of hamstring injury as predictors for recurrent injury. J Orthop Sports Phys Ther 2006;36:215-24.

28 Gibbs NJ, Cross TM, Cameron M, et al. The accuracy of MRI in predicting recovery and recurrence of acute grade one hamstring muscle strains within the same season in Australian Rules football players. I Sci Med Sport Sports Med Aust 2004;7:248-58.
29 Koulouris G, Connell DA, Brukner P, et al. Magnetic resonance imaging parameters for assessing risk of recurrent hamstring injuries in elite athletes. Am I Sports Med 2007:35:1500-6.

30 Bahr R, Holme I. Risk factors for sports injuries-a methodological approach. $\mathrm{Br} J$ Sports Med 2003:37:384-92.

31 Creighton DW, Shrier I, Shultz R, et al. Return-to-play in sport: a decision-based model. Clin J Sport Med 2010;20:379-85. 

predict hamstring re-injury, but baseline MRI findings do not

Robert-Jan De Vos, Gustaaf Reurink, Gert-Jan Goudswaard, Maarten H Moen, Adam Weir and Johannes L Tol

Br J Sports Med 2014 48: 1377-1384 originally published online July 18, 2014

doi: 10.1136/bjsports-2014-093737

Updated information and services can be found at:

http://bjsm.bmj.com/content/48/18/1377

These include:

Supplementary Supplementary material can be found at:

Material http://bjsm.bmj.com/content/suppl/2014/07/19/bjsports-2014-093737. DC1.html

References This article cites 30 articles, 17 of which you can access for free at: http://bjsm.bmj.com/content/48/18/1377\#BIBL

Email alerting Receive free email alerts when new articles cite this article. Sign up in the service box at the top right corner of the online article.

\begin{tabular}{|c|c|}
\hline $\begin{array}{l}\text { Topic } \\
\text { Collections }\end{array}$ & Articles on similar topics can be found in the following collections \\
\hline Collections & BJSM Reviews with MCQs (66) \\
\hline
\end{tabular}

\section{Notes}

To request permissions go to:

http://group.bmj.com/group/rights-licensing/permissions

To order reprints go to:

http://journals.bmj.com/cgi/reprintform

To subscribe to BMJ go to:

http://group.bmj.com/subscribe/ 